\title{
Modelling the skeletal muscle injury recovery using in vivo contrast-enhanced micro-CT: a proof-of-concept study in a rat model
}

Bruno Paun ${ }^{1}$, Daniel García Leon ${ }^{1}$, Alex Claveria Cabello ${ }^{1}$, Roso Mares Pages ${ }^{1}$, Elena de la Calle Vargas ${ }^{1}$, Paola Contreras Muñoz ${ }^{2,3}$, Vanessa Venegas Garcia ${ }^{2,3}$, Joan Castell-Conesa ${ }^{1}$, Mario Marotta Baleriola ${ }^{2,3}$ and Jose Raul Herance Camacho ${ }^{1 *}$

\begin{abstract}
Background: Skeletal muscle injury characterisation during healing supports trauma prognosis. Given the potential interest of computed tomography (CT) in muscle diseases and lack of in vivo CT methodology to image skeletal muscle wound healing, we tracked skeletal muscle injury recovery using in vivo micro-CT in a rat model to obtain a predictive model.

Methods: Skeletal muscle injury was performed in 23 rats. Twenty animals were sorted into five groups to image lesion recovery at $2,4,7,10$, or 14 days after injury using contrast-enhanced micro-CT. Injury volumes were quantified using a semiautomatic image processing, and these values were used to build a prediction model. The remaining 3 rats were imaged at all monitoring time points as validation. Predictions were compared with BlandAltman analysis.

Results: Optimal contrast agent dose was found to be $20 \mathrm{~mL} / \mathrm{kg}$ injected at $400 \mu \mathrm{L} / \mathrm{min}$. Injury volumes showed a decreasing tendency from day $0\left(32.3 \pm 12.0 \mathrm{~mm}^{3}\right.$, mean \pm standard deviation) to day $2,4,7,10$, and 14 after injury $\left(19.6 \pm 12.6,11.0 \pm 6.7,8.2 \pm 7.7,5.7 \pm 3.9\right.$, and $4.5 \pm 4.8 \mathrm{~mm}^{3}$, respectively). Groups with single monitoring time point did not yield significant differences with the validation group lesions. Further exponential model training with single follow-up data $\left(R^{2}=0.968\right)$ to predict injury recovery in the validation cohort gave a predictions root mean squared error of $6.8 \pm 5.4 \mathrm{~mm}^{3}$. Further prediction analysis yielded a bias of 2.327 .
\end{abstract}

Conclusion: Contrast-enhanced CT allowed in vivo tracking of skeletal muscle injury recovery in rat.

Keywords: Muscle (skeletal), Muscular diseases, Rats, Tomography (x-ray computed), Wound healing

\footnotetext{
* Correspondence: raul.herance@vhir.org

'Medical Molecular Imaging Group, Vall d'Hebron Research Institute (VHIR),

CIBER-BBN, CIBBIM-Nanomedicine, ISCIII, Hospital Universitari Vall d'Hebron,

Universitat Autònoma de Barcelona (UAB), Passeig de la Vall d'Hebron

119-129, 08035 Barcelona, Spain

Full list of author information is available at the end of the article
}

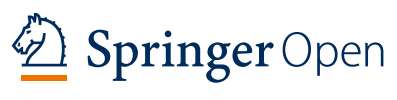

(c) The Author(s). 2020 Open Access This article is licensed under a Creative Commons Attribution 4.0 International License, which permits use, sharing, adaptation, distribution and reproduction in any medium or format, as long as you give appropriate credit to the original author(s) and the source, provide a link to the Creative Commons licence, and indicate if changes were made. The images or other third party material in this article are included in the article's Creative Commons licence, unless indicated otherwise in a credit line to the material. If material is not included in the article's Creative Commons licence and your intended use is not permitted by statutory regulation or exceeds the permitted use, you will need to obtain permission directly from the copyright holder. To view a copy of this licence, visit http://creativecommons.org/licenses/by/4.0/. 


\section{Key points}

- Contrast-enhanced micro-computed tomography showed applicability in monitoring skeletal muscle injury recovery.

- Highest accuracy in lesion recovery predictions was obtained on day 14 after injury.

- We provided a proof of concept to track skeletal muscle healing stages using CT-derived volumebased lesion recovery.

\section{Background}

Skeletal muscle (SM) represents about $40 \%$ of the body mass and is formed by contractile multinucleated muscle fibres as a result from myoblasts fusion. Injuries in SM may come from diverse events, including direct trauma from muscle contusions, lacerations and strains or indirect trauma from degenerative diseases like muscular dystrophy, among others. Professional athletes are the most affected by SM injuries, representing from 10 to $55 \%$ of all injuries in this population [1,2]. Moreover, the type, severity, size and location of the injury make inference of recovery and rehabilitation times challenging, thus directly affecting prognosis of lay-off times [3].

In mammals, SM has the ability to regenerate in response to injury [4]. This process can be divided into three phases [1, 4-6]: (1) degeneration, characterised by muscle fibres rupture and necrosis, formation of hematoma and inflammatory cell reaction; (2) regeneration, characterised by phagocytosis of the damaged tissue, satellite cells (SM stem cells) activation and angiogenesis; and (3) remodelling, characterised by the maturation and reorganisation of regenerated muscle fibres, recovery of muscle function together with fibrosis and scar tissue formation. The new vascular network emerges from surviving trunks of the blood vessels into the injured region providing means for oxygen delivery and nutrients necessary to recover mature myofibres $[1,7]$.

To characterise in vivo muscle injury, both magnetic resonance imaging (MRI) and ultrasound are wellestablished imaging approaches in hospitals [8]. However, there is a knowledge gap regarding the approaches to monitor and quantify SM injury using computed tomography $(\mathrm{CT})$, which plays an important role in tissue density characterisation in muscle diseases [9]. Along with its application in whole-body scans, CT is also part of hybrid scanners as single-photon computed tomography/CT or positron emission tomography (PET)/CT where functional studies can be conducted to monitor SM lesion healing.

To the best of our knowledge, there are no studies where CT has been used for in vivo tracking of SM injury. There are several studies in the literature $[7,10]$, where the authors use ex vivo contrast-enhanced micro$\mathrm{CT}$ to quantify underlying angiogenesis in the process of wound healing. Therefore, the lack of methodologies allowing in vivo assessment of SM recovery was the main motivation of the present work.

Consequently, we aimed to (i) present a new methodology to image and quantify in vivo SM recovery by contrast-enhanced micro-CT means and (ii) propose a proof-of-principle study of a SM recovery prediction model.

\section{Methods \\ Animal preparation}

The study was approved by the Local Animal Ethics Committee and was performed in accordance with Spanish (RD 53/2013) and European (2010/63/UE) legislation. Wistar male adult rats $(n=27)$, weighing approximately $425 \mathrm{~g}$ each $(425.4 \pm 43 \mathrm{~g})$, were housed at $22-24{ }^{\circ} \mathrm{C}$, maintained on a 12 -h light/dark cycle, and used for the experiments. Water and food were given ad libitum during the experiments. Anaesthesia was induced with $4 \%$ isoflurane (Aerrane, Baxter Healthcare, Deerfield, IL, USA) and maintained with $1.5-2.0 \%$ isoflurane in $\mathrm{O}_{2}$ at $1 \mathrm{~L} / \mathrm{min}$ during the whole study. A 22-gauge intravenous cannula (Introcan Safety ${ }^{\circ}$ IV Catheter, 22G, B. Braun, Melsungen, Germany) was placed in the tail vein for iopamidol (iopamiro $370 \mathrm{mg} / \mathrm{mL}$, Bracco Imaging, Milan, Italy) administration as contrast agent (CA) by means of an infusion pump (Harvard apparatus PHD 2000 Infusion, Holliston, MA, USA). Post-surgically, $250 \mathrm{~mL}$ of water with $10 \%$ of buprenorphine (buprecare $0.3 \mathrm{mg} /$ $\mathrm{mL}$, Divasa Farmavic, Barcelona, Spain) was given to all operated animals.

\section{Contrast agent dose optimisation}

Optimal CA dose to distinguish between injury and neighbouring tissue was first determined by performing a contrast enhancement study in a separate set of experiments including five rats. These were placed in the micro-CT bed, and five different infusion rates (100, 200, 300, 400, and $500 \mu \mathrm{L} / \mathrm{min}$ ) were tested for $20 \mathrm{~min}$ to obtain different contrast-to-noise ratios (CNR) in acquired images. Imaging parameters were as follows: field of view $40 \mathrm{~mm}$, voxel size $80 \mu \mathrm{m}$ (isotropic), $\mathrm{kVp} \mathrm{90,}$ tube current $200 \mu \mathrm{A}$ and exposure time $120 \mathrm{~s}$. Images were acquired approximately every $2.5 \mathrm{~min}$, resulting in total of 9 images per rat. Contrast enhancement was assessed in the femoral artery and neighbouring tissue by delineating circular volumes of interest of $1 \mathrm{~mm}$ in diameter, obtaining their mean density and standard deviation values. From these, we calculated CNR using following formula [11]: 


$$
C N R=\frac{\left|\mu_{\mathrm{A}}-\mu_{\mathrm{T}}\right|}{\sqrt{\sigma_{\mathrm{A}}^{2}+\sigma_{\mathrm{T}}^{2}}},
$$

where $\mu_{\mathrm{A}}$ and $\mu_{\mathrm{T}}$ represent mean density values of volumes of interest in femoral artery and tissue area, respectively, and $\sigma_{\mathrm{A}}$ and $\sigma_{\mathrm{T}}$ represent their standard deviations. Optimal infusion rate providing adequate image contrast was set following the Rose criterion (CNR > 5), as previously described [12].

After CA administration, the pump was stopped, and micro-CT images were acquired. One millilitre of saline solution was injected after image acquisition was finished.

\section{Skeletal muscle injury model}

For the injury model, we used a recently developed surgically induced SM injury rat model proposed by Contreras-Muñoz et al [6]. Briefly, 23 anaesthetised rats were immobilised by the fixation of tail and extremities with adhesive strips to a styrofoam surface exposing the ventral side of the right crus. Skeletal traumatic muscle injuries were induced in the rat medial gastrocnemius muscle by a 18 -gauge biopsy needle Bard $^{\circ}$ Monopty $^{\circ}$ Disposable Core Biopsy Instrument, Bard Biopsy Systems, Tempe, USA) with a 0.84-mm inner diameter. Transversal biopsy procedure was performed at the muscle-tendon junction level of the left leg medial gastrocnemius muscle $(3 \mathrm{~mm}$ from the start of muscletendon junction and $2 \mathrm{~mm}$ in depth). Immediately after muscle injury, a cannula (Introcan Safety ${ }^{\circ}$ IV Catheter, 22G, B. Braun, Melsungen, Germany) was introduced within the injury in order to proceed with the micro-CT imaging protocol.

\section{Micro-CT}

Micro-CT studies were performed using a Quantum FX micro-CT scanner (PerkinElmer, Hopkinton, MA, USA). Rats $(n=23)$ were positioned on their right side in a bed, and their left leg was immobilised to minimise possible involuntary movement and motion-related artefacts. During the scans, rats were kept under anaesthesia, and CA was administered for $20 \mathrm{~min}$ as described above. Two images were acquired at the same day when muscle injury was induced: first an image of the injury with the cannula at $14 \mathrm{~min}$ after the beginning of CA infusion for location purposes, and then, after removing the cannula from the injury, a second image was acquired at $20 \mathrm{~min}$ right after stopping the CA injection. Again, $1 \mathrm{~mL}$ of saline solution was injected after image acquisition was finished to ease contrast clearance.

To image lesion recovery at different time points, 20 rats were sorted into 5 different groups $(n=4$ per group) according to the follow-up day at 2, 4, 7, 10, or 14 days after injury, respectively. Additional 3 rats were imaged at all mentioned follow-up days as validation. High-resolution images were acquired so that the centre of the field of view was aligned with the middle of the fibula in sagittal and coronal planes, thus covering the maximal region of the left limb containing the injury. Experimental imaging parameters were field of view $40 \mathrm{~mm}$, voxel size $80 \mu \mathrm{m}$ (isotropic), $\mathrm{kVp}$ 90, tube current $200 \mu \mathrm{A}$ and exposure time $270 \mathrm{~s}$. All images were reconstructed using a filtered back-projection approach with a Ram-Lak filter, including a ring reduction algorithm [13].

\section{Image processing}

Greyscale values between image datasets were normalised by an histogram matching algorithm implemented in the Insight Toolkit [14]. All image datasets in the study were normalised by matching their histograms to the reference image histogram, which was chosen to be the injury image of rat 1 at day 0 . Adapted normalised image datasets were then filtered in Fiji [15] using a non-local means denoising filter [16, 17]. Noise standard deviation was set to 2 with smoothing factor set to 1 . Contrast-enhanced anatomy, together with highintensity anatomy like bones, was segmented from denoised images using thresholding based on Renyi entropy [18] with min and max values set to 77 and 255, respectively. These values were experimentally derived from denoised images to ease the injury segmentation process. All segmented contrast-enhanced anatomy images were then manually processed in three-dimensional slicer (version 4.9.0) [19]. The injury was further segmented from contrast-enhanced anatomy mask, excluding surrounding adipose tissue and vasculature. Once the refined SM injury mask was obtained, injury volume was calculated as the mask voxel number multiplied by voxel volume.

\section{Lesion recovery prediction model}

Single follow-up day cohort data $(n=20)$ were used to feed an exponential model of SM injury recovery. The generalised exponential model used was $V t=V_{0} \times \exp$ $(b \times t)$, where $t$ is the corresponding post-injury time (days), $V_{0}$ is the initial injury size $\left(\mathrm{mm}^{3}\right)$ and $b$ is the healing rate $\left(\right.$ days $\left.^{-1}\right)$. Model was trained using the least absolute residual method available in Matlab (The Mathworks Inc., Natick, MA, USA), quantifying both $R^{2}$ and root mean squared error. Afterwards, injury volumes were predicted in the validation cohort $(n=3)$ at all monitored post-injury time points.

\section{Statistical analysis}

Data are expressed as mean and standard deviation. Injury volumes and model predictions were compared using paired $t$ test and Bland-Altman analysis after 
testing for normality. All statistical analyses were performed using the Graphpad Prism software (Graphpad Software Inc., San Diego, CA, USA). A $p$ value below 0.05 was considered as statistically significant.

\section{Results}

\section{Optimal contrast agent dose}

Prior to tracking injury healing by micro-CT, analysis of the influence of CA dose in image contrast was performed to determine optimal infusion rate. Figure 1 shows obtained CNR curves for each tested infusion rate. Since cutoff criterion was set at CNR over 5 after $20 \mathrm{~min}$ of infusion, contrast injection rates of 400 and $500 \mu \mathrm{L} / \mathrm{min}$ were the only ones satisfying this requirement. However, to reduce the amount of CA injected to animals and to avoid possible side effects, $400 \mu \mathrm{L} / \mathrm{min}$ was chosen as optimal rate and was used in following study, resulting in total administered amount of contrast of $20 \mathrm{~mL} / \mathrm{kg}$ per rat.

\section{In vivo SM lesion tracking}

In Fig. 2, one example of SM lesion healing in short-axis view monitored at all post-injury time points is presented. As shown, lesion healing process remains rather active up to 2 weeks after injury. This was further quantified in Fig. 3, sorting lesion volumes according to the day injury was imaged. The mean initial lesion size $(n=$ 23) was $32.3 \pm 12.0 \mathrm{~mm}^{3}$. As expected, lesion healing process yielded decreasing injury volumes $(n=7)$ at day $2\left(19.6 \pm 12.6 \mathrm{~mm}^{3}\right)$, day $4\left(11.0 \pm 6.7 \mathrm{~mm}^{3}\right)$, day $7(8.2$ $\left.\pm 7.7 \mathrm{~mm}^{3}\right)$, day $10\left(5.7 \pm 3.9 \mathrm{~mm}^{3}\right)$, and day $14(4.47 \pm$ $4.8 \mathrm{~mm}^{3}$ ) post-injury. Additionally, groups with a single monitored time point after injury ( 5 time points, $n=4$ each) were compared to the validation cohort $(n=3)$ to support this decreasing tendency. Indeed, lesion volumes did not show any statistically significant differences between single time point groups and validation group at day $0\left(34.0 \pm 11.9\right.$ versus $\left.21.4 \pm 3.8 \mathrm{~mm}^{3}, p=0.088\right)$, day $2\left(22.1 \pm 14.5\right.$ versus $\left.16.3 \pm 11.7 \mathrm{~mm}^{3}, p=0.598\right)$, day $4\left(11.3 \pm 5.3\right.$ versus $\left.10.7 \pm 9.6 \mathrm{~mm}^{3}, p=0.915\right)$, day $7\left(9.2 \pm 9.7\right.$ versus $\left.7.0 \pm 5.9 \mathrm{~mm}^{3}, p=0.744\right)$, day $10(6.4$ \pm 4.5 versus $\left.4.7 \pm 3.8 \mathrm{~mm}^{3}, p=0.622\right)$ and at day 14 ( $(3.5$ \pm 3.0 versus $\left.5.8 \pm 0.4 \mathrm{~mm}^{3}, p=0.584\right)$, respectively.

\section{Injury volume predictions}

Model training with single follow-up data yielded a correlation of $R^{2}=0.968$, with root-mean-square error $=$ $1.77 \mathrm{~mm}^{3}$. This was further tested with the validation cohort $(n=3)$ at all monitored time points (days 2, 4, 7, 10 , and 14 after injury). Mean root-mean-square error in predictions was $6.8 \pm 5.4 \mathrm{~mm}^{3}$. As presented in the Bland-Altman plot (Fig. 4), overall predictions yielded a bias of 2.3 (limits of agreement at 95\%, -13.5 and 18.1). The exponential model better performed at low average values, offering higher accuracy for nearly healed injury time points.

\section{Discussion}

In this proof-of-concept study, we assessed the in vivo CT applicability to track the progression of SM injury recovery. For this purpose, we previously defined a contrast enhancement CT protocol in order to guarantee proper differentiation between injury and healthy tissue. After quantifying injury volumes during follow-up to track injury recovery, an exponential prediction model of injury recovery has been proposed to mimic the observed decreasing tendency during healing. Although simple, the model offered a higher accuracy for late follow-up time points than that for early time points when the lesion is nearly recovered.

Regarding the contrast enhancement protocol, we found a CA volume of $20 \mathrm{~mL} / \mathrm{kg}$ that was necessary to

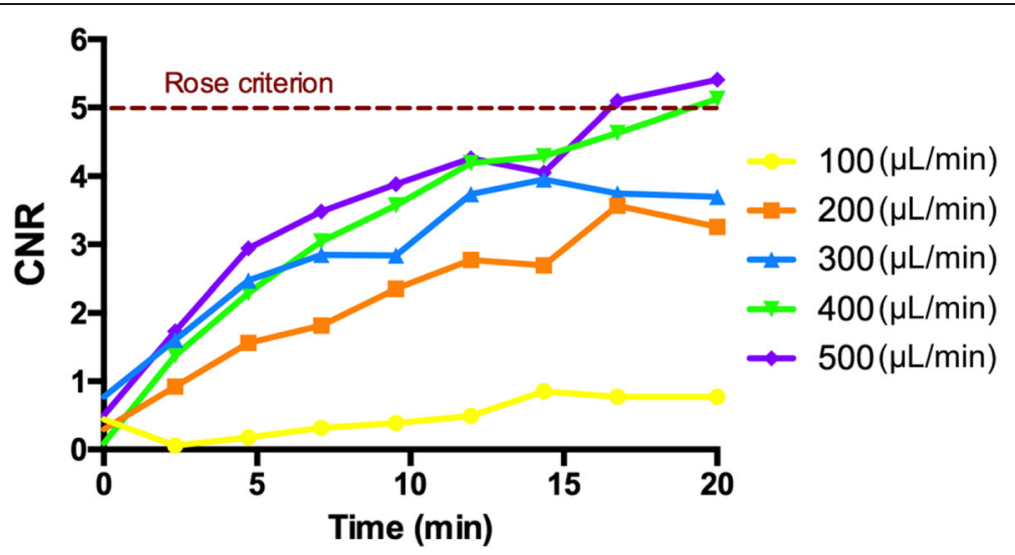

Fig. 1 Contrast-to-noise ratio quantification for several infusion rates. Tested contrast injection rates from 100 to $500 \mu \mathrm{L} / \mathrm{min}$ (yellow), with an overall administration time of $20 \mathrm{~min}$. The red dashed line defines the applied cutoff criterion to determine the optimal infusion rate. CNR Contrast-to-noise ratio 


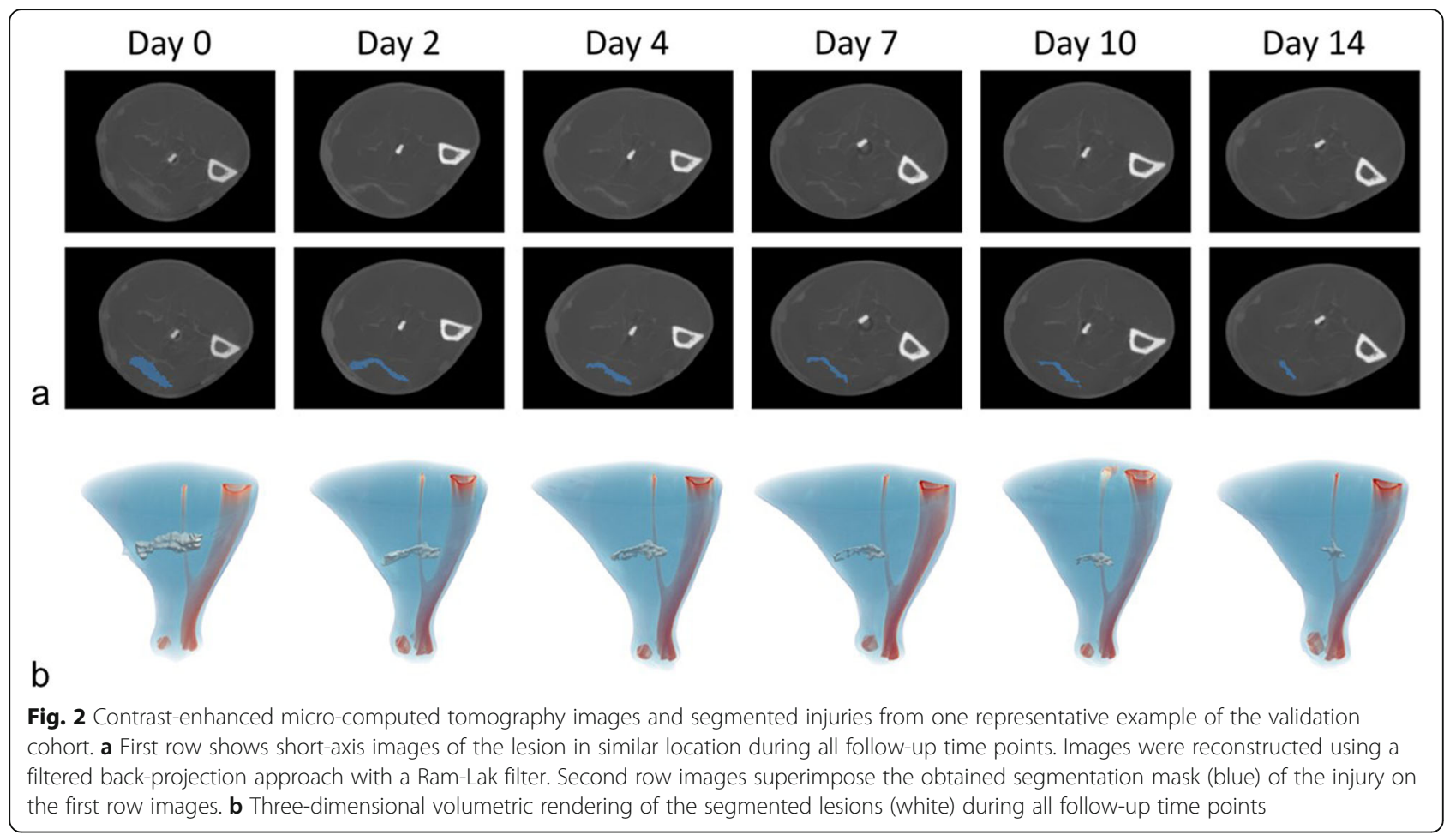

obtain a proper CNR on CT images. Injecting more contrast may cause unnecessary side effects in rats without significant increasing image contrast. In addition, the proposed injection rate of $400 \mu \mathrm{L} / \mathrm{min}$ was found to be the best rate allowing a proper biodistribution of the $\mathrm{CA}$ without causing any side effect in rats. This experimentally determined CA amount was in agreement with guidelines about intravenous administration volumes for rats reported by Diehl et al [20].

The murine model of skeletal muscle injury used in this study has been previously described in detail [6]. As

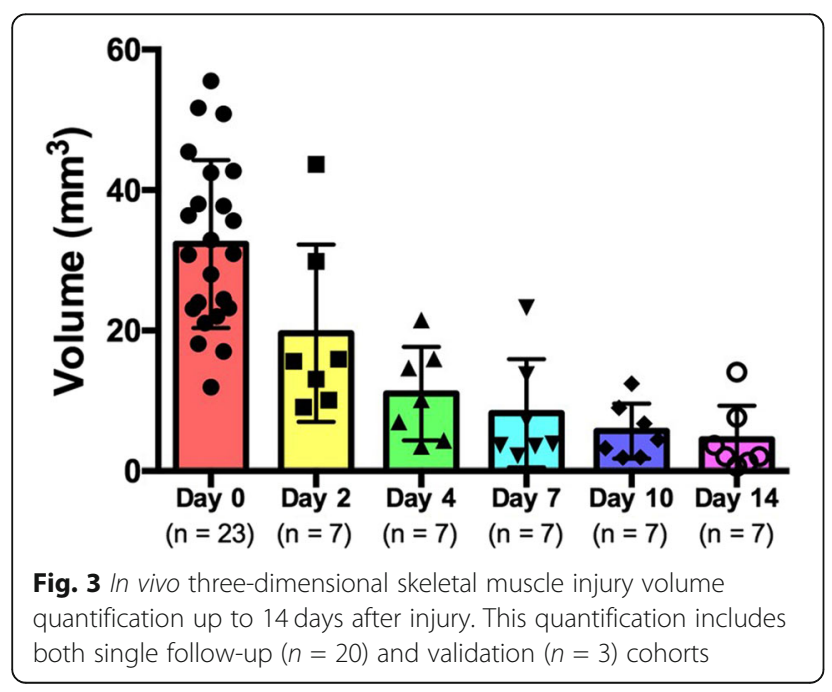

previously reported, this injury model well reproduces the human muscle strain injuries observed in professional athletes. This was characterised by means of histology and MRI (standard of care in muscle lesion tracking), showing the healing capacity observed in animal models of spontaneous skeletal muscle injury as the dystrophic $\mathrm{mdx}$-mice [21]. Although similar looking skeletal muscle injuries on MRI at the same anatomical site, different healing rates have been observed as the lesion nature varies (either high-speed running or overstretching) in athletes [22].

This study focused on the applicability of in vivo CT imaging to track SM lesion recovery. However, established imaging techniques for prognosis and muscle repair monitoring in the clinics are both MRI and ultrasound [8]. The latter is normally considered to be first choice for clinical diagnosis due to its low price and fast applicability, but MRI is preferred when detailed characterisation of injury is required [23]. Recently, Todeschini et al. [24] reported a series of 39 professional soccer players to study pubalgia imaging features with both ultrasound and MRI. Interestingly, from 9 individuals with a positive MRI scan regarding lesions of the common aponeurosis of the rectus abdominis/adductor longus muscles, only 4 yielded a positive ultrasound scan. MRI has been positioned as a widely used technique in muscle trauma evaluation, playing an increasingly role in grading injury severity and guiding return to play in the injured athlete [25]. Nonetheless, this 


\section{Bland-Altman analysis of recovery predictions}

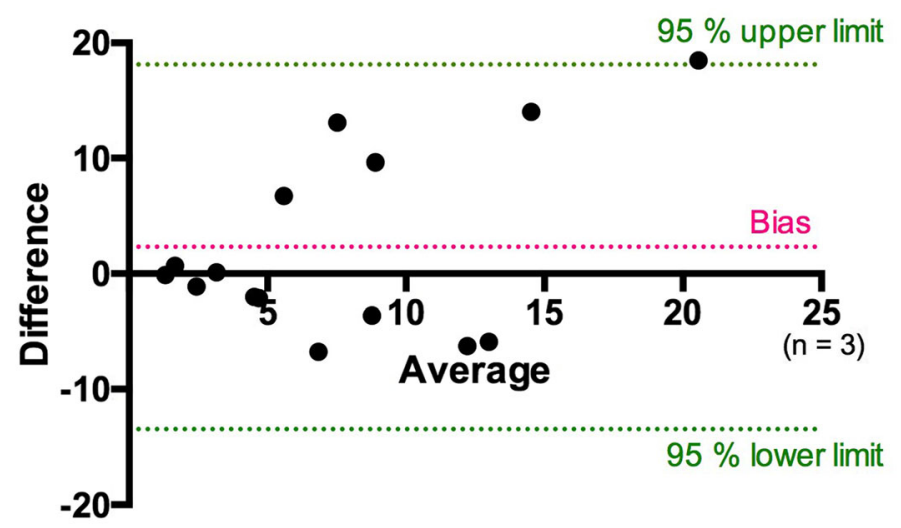

Fig. 4 Bland-Altman analysis of the predicted injury volumes in the validation cohort. Predictions were compared to the injury volumes during all follow-up time points. Red dotted line corresponds to the bias, with corresponding lower and upper limits of agreement at $95 \%$ represented with green dotted lines

technique does not offer any information regarding tissue dynamics when healing as a counterpart. Leaning on hybrid approaches using PET/CT or PET/MRI scans, this may be of relevance to study SM lesion healing dynamics in athletes.

There are just a few studies using PET/CT to assess muscle injury. For instance, Carter et al [26] studied metabolic changes related to thermally induced muscle injury in rabbits using 2-deoxy-2-[fluorine-18]fluoro-Dglucose $\left({ }^{18}\right.$ F-fluorodeoxyglucose, ${ }^{18}$ F-FDG $)$ PET. Xie et al [27] showed that mice with muscle injury induced by electroporation show increased uptake of ${ }^{64} \mathrm{Cu}$, thus demonstrating ${ }^{64} \mathrm{Cu}-\mathrm{Cl}_{2}$ PET/CT as a new molecular imaging technique for skeletal muscle. Recently, Pervaiz et al [28] showed that ${ }^{18} \mathrm{~F}$-FDG PET/CT can be used for imaging inflammation and muscle injury caused by cholesterol crystal emboli. However, these studies focused on the use of radiopharmaceuticals to assess a specific physiological disorder that cannot explain the whole SM recovery. Merging these findings with in vivo contrastenhanced CT quantifying muscle recovery, both imaging techniques may offer enriched lesion healing structural and functional information, yielding a more accurate injury prognosis, among other uses.

Although simple, this study also presented an exponential model of muscle recovery as a proof of concept. This model relied on quantified injury volumes through time to mimic the observed decreasing tendency derived from two-dimensional histological analyses in previous studies [6]. Yielded predictions were accurate when lesion volumes were nearly recovered but differed more at few days after injury. One way to better predict lesion recovery at all monitored time points could be to detect additional lesion features via convolutional neural network $(\mathrm{CNN})$ models. These deep learning models can automatically learn hierarchical features from raw images to classify these according to a training classification [29]. For instance, Zhang and colleagues [30] presented a CNN model to classify soft tissue sarcoma grading using both CT and MR images from a series of 51 patients, offering great performance in this task. In this matter, CNNs could definitely be applied to track skeletal muscle injury healing, supporting current trauma prognosis.

This study has several limitations. First, there is a limited number of rats included in the validation cohort. Although tracking full progression of the injury recovery in all animals would have been desirable, no statistical differences were observed between the single follow-up day and the validation cohorts. Second, no histological analysis of the lesions was provided. Conversely, reported three-dimensional SM lesion characterisation data are consistent with previously reported histological results. Regarding lesion recovery, a direct association to professional athlete lesions cannot be established as the lesion nature varies from the surgically induced one in the rat model. Also, in vivo SM lesion tracking cannot be directly extrapolated to human studies. Indeed, the amount of iodine-based contrast agent used in our experiments exceeds the maximum recommended dose for contrast enhancement studies included in current clinical guidelines. To overcome the low soft tissue contrast problem during SM injury monitoring, early injury stages may offer a good starting point for tracking. For instance, both oedema and inflammatory processes in clinical contrast-enhanced CT images could serve as initial landmarks to ease injury segmentation process. Adapting this prior segmentation to subsequent postinjury examinations leaning on anatomical references detection, recovery predictions and the expert 
knowledge to refine lesion border delineation accordingly may offer similar outcomes as the ones we presented from animal experiments. Of note, acquired $z$ axis resolution in conventional CT scanners $(\sim 3 \mathrm{~mm})$ might hinder this task, so detailed studies would be required to minimise the use of CA. Altogether, additional in vivo lesion tracking using contrast-enhanced $\mathrm{CT}$ in large animal models first, and then in patient studies, would be required to further validate these results.

In conclusion, our study showed that contrastenhanced CT can allow in vivo tracking of SM healing after injury, presenting a proof of concept of an exponential recovery model, in a rat model.

\section{Abbreviations \\ CA: Contrast agent; CNN: Convolutional neural network; CNR: Contrast-to- noise ratio; CT: Computed tomography; FDG: Fluorodeoxyglucose; MRI: Magnetic resonance imaging; PET: Positron emission tomography; SM: Skeletal muscle}

\section{Authors' contributions}

Conceptualisation and formulation of the ideas presented in this manuscript were done by JRHC, BP, and ACC. Data curation and statistical, mathematical, computational, or other formal analysis of the data were performed by BP and ECV. Acquisition of the financial support for the project leading to this publication was done by JRHC, JCC, and MMB. Research and investigation process, more precisely experiments and data/evidence collection were performed by BP, ACC, RMP, WG, PCM, JCC, and MMB. Development and design of methodology and creation of models were done by BP, PCM, and JRHC. Provision of study materials, reagents, materials, patients, laboratory samples, animals, instrumentation, computing resources, or other analysis tools was performed by JRHC, ACC, RMP, and WG. Programming, implementation of the computer code and supporting algorithms and testing of existing code components were performed by BP and ECV. Validation of the results/experiments and other research outputs was done by JRHC, BP. JRHC, BP, ACC, MMB, and JCC contributed in writing, reviewing and editing of the manuscript. All authors read and approved the final manuscript.

\section{Funding}

This study has received funding by European Commission (Horizon 2020), the Carlos III Health Institute and the European Regional Development Fund (PI16/02064) and AGAUR from Generalitat de Catalunya (2017SGR1303).

\section{Availability of data and materials}

The datasets used and/or analysed during the current study are available from the corresponding author on reasonable request.

\section{Ethics approval}

Approval from the institutional animal care committee was obtained.

\section{Consent for publication}

Not applicable.

\section{Competing interests}

The authors declare that they have no competing interests.

\section{Author details}

'Medical Molecular Imaging Group, Vall d'Hebron Research Institute (VHIR), CIBER-BBN, CIBBIM-Nanomedicine, ISCIII, Hospital Universitari Vall d'Hebron, Universitat Autònoma de Barcelona (UAB), Passeig de la Vall d'Hebron 119-129, 08035 Barcelona, Spain. ${ }^{2}$ Health \& Biomedicine division, Leitat Technological Center, 2. C/ Pallars, 179-185, 08005 Barcelona, Spain. ${ }^{3}$ Bioengineering, Cell therapy and Surgery in Congenital Malformations Laboratory, Vall d'Hebron Research Institute (VHIR), Hospital Universitari Vall d'Hebron, Universitat Autònoma de Barcelona (UAB), Passeig de la Vall d'Hebron 119-129, 08035 Barcelona, Spain.
Received: 8 November 2019 Accepted: 22 April 2020

Published online: 03 June 2020

\section{References}

1. Järvinen $T A$, Järvinen $T L$, Kääriäinen $M$, Kalimo $H$, Järvinen $M$ (2005) Muscle injuries: biology and treatment. Am J Sports Med 33:745-764 https://doi. org/10.1177/0363546505274714

2. Garg K, Corona BT, Walters TJ (2015) Therapeutic strategies for preventing skeletal muscle fibrosis after injury. Front Pharmacol 6. https://doi.org/10. 3389/fphar.2015.00087

3. Ekstrand J, Healy JC, Waldén M, Lee JC, English B, Hägglund M (2012) Hamstring muscle injuries in professional football: the correlation of MRI findings with return to play. Br J Sports Med 46:112-117 https://doi.org/10. 1136/bjsports-2011-090155

4. Laumonier T, Menetrey J (2016) Muscle injuries and strategies for improving their repair. J Exp Orthop 3 https://doi.org/10.1186/s40634-016-0051-7

5. Chargé SBP, Rudnicki MA (2004) Cellular and molecular regulation of muscle regeneration. Physiol Rev 84:209-238 https://doi.org/10.1152/physrev.00019. 2003

6. Contreras-Muñoz P, Fernández-Martín A, Torrella R et al (2016) A new surgical model of skeletal muscle injuries in rats reproduces human sports lesions. Int J Sports Med 37:183-190 https://doi.org/10.1055/s-0035-1555933

7. Singh DP, Barani Lonbani Z, Woodruff MA, Parker TJ, Steck R, Peake JM (2017) Effects of topical icing on inflammation, angiogenesis, revascularization, and myofiber regeneration in skeletal muscle following contusion injury. Front Physiol 8:1-15 https://doi.org/10.3389/fphys.2017. 00093

8. Slavotinek JP (2010) Muscle injury: the role of imaging in prognostic assignment and monitoring of muscle repair. Semin Musculoskelet Radiol 14:194-200 https://doi.org/10.1055/s-0030-1253160

9. Engelke K, Museyko O, Wang L, Laredo JD (2018) Quantitative analysis of skeletal muscle by computed tomography imaging — state of the art. J Orthop Transl 15:91-103 https://doi.org/10.1016/j.jot.2018.10.004

10. Urao N, Okonkwo UA, Fang MM, Zhuang ZW, Koh TJ, DiPietro LA (2016) MicroCT angiography detects vascular formation and regression in skin wound healing. Microvasc Res 106:57-66 https://doi.org/10.1016/j.physbeh. 2017.03.040

11. Demirturk Kocasarac $H$, Helvacioglu Yigit D, Bechara B, Sinanoglu A, Noujeim M (2016) Contrast-to-noise ratio with different settings in a CBCT machine in presence of different root-end filling materials: an in vitro study. Dentomaxillofacial Radiol 45. https://doi.org/10.1259/dmfr.20160012

12. Burgess AE (1999) The Rose model, revisited. J Opt Soc Am A Opt Image Sci Vis 16:633 https://doi.org/10.1364/josaa.16.000633

13. Jang SY, Kim HK, Youn H, Cho S, Cunningham IA (2017) Fourier analysis of noise characteristics in cone-beam microtomography laboratory scanners. IEEE Trans Biomed Eng 64:173-183 https://doi.org/10.1109/TBME.2016. 2552496

14. Yoo TS, Ackerman MJ, Lorensen WE et al (2002) Engineering and algorithm design for an image processing API: a technical report on ITK - The Insight Toolkit. In: Studies in Health Technology and Informatics. IOS Press, pp 586592 https://doi.org/10.3233/978-1-60750-929-5-586

15. Schindelin J, Arganda-Carreras I, Frise E et al (2012) Fiji: An open-source platform for biological-image analysis. Nat Methods 9:676-682 https://doi. org/10.1038/nmeth.2019

16. Buades A, Coll B, Morel J-M (2011) Non-local means denoising. Image Processing On Line 1:208-212 https://doi.org/10.5201/ipol.2011.bcm_nlm

17. Darbon J, Cunha A, Chan TF, Osher S, Jensen GJ (2008) Fast nonlocal filtering applied to electron cryomicroscopy. IEEE:1331-1334 https://doi.org/ $10.1109 /$ ISBI.2008.4541250

18. Wang S, Jia H, Peng X (2020) Modified salp swarm algorithm based multilevel thresholding for color image segmentation. Math Biosci Eng 17 700-724 https://doi.org/10.3934/mbe.2020036

19. Fedorov A, Beichel R, Kalpathy-Cramer J et al (2012) 3D Slicer as an image computing platform for the quantitative imaging network. Magn Reson Imaging 30:1323-1341 https://doi.org/10.1016/.mri.2012.05.001

20. Diehl K, Hull R, Morton D et al (2001) A good practice guide to the administration of substances and removal of blood, including routes and volumes. J Appl Toxicol 23:15-23 https://doi.org/10.1002/jat.727

21. Marotta M, Sarria Y, Ruiz-Roig C, Munell F, Roig-Quilis M (2007) Laser microdissection-based expression analysis of key genes involved in muscle 
regeneration in mdx mice. Neuromuscul Disord 17:707-718 https://doi.org/ 10.1016/.j.nmd.2007.05.007

22. Askling CM, Malliaropoulos N, Karlsson J (2012) High-speed running type or stretching-type of hamstring injuries makes a difference to treatment and prognosis. Br J Sports Med 46:86-87 https://doi.org/10.1136/bjsports-2011090534

23. Järvinen $T A H$, Järvinen $T L N$, Kääriäinen $M$ et al (2007) Muscle injuries: optimising recovery. Best Pract Res Clin Rheumatol 21:317-331 https://doi. org/10.1016/j.berh.2006.12.004

24. Todeschini K, Daruge P, Bordalo-Rodrigues M, Pedrinelli A, Busetto AM (2019) Imaging assessment of the pubis in soccer players. Rev Bras Ortop 54:118-127 https://doi.org/10.1016/j.rbo.2017.12.012

25. Flores DV, Mejía Gómez C, Estrada-Castrillón M, Smitaman E, Pathria MN (2018) MR imaging of muscle trauma: anatomy, biomechanics, pathophysiology, and imaging appearance. Radiographics 38:124-148 https://doi.org/10.1148/rg.2018170072

26. Carter EA, Tompkins RG, Hsu H et al (1997) Metabolic alterations in muscle of thermally injured rabbits, measured by positron emission tomography. Life Sci 61:39-44 https://doi.org/10.1016/S0024-3205(97)00355-X

27. Xie F, Cai H, Peng F (2017) CuCl 2 PET / CT imaging of mouse muscular injury induced by electroporation. Am J Nucl Med Mol Imaging 7:33-39 Retrieved from http://www.ainmmi.us/

28. Pervaiz MH, Durga S, Janoudi A, Berger K, Abela GS (2018) PET/CTA detection of muscle inflammation related to cholesterol crystal emboli without arterial obstruction. J Nucl Cardiol 25:433-440 https://doi.org/10 1007/s12350-017-0826-y

29. Huang L, Gong ZY, Jun YT (2019) Skin lesion segmentation using object scale-oriented fully convolutional neural networks. Signal Image Video Process 13:431-438 https://doi.org/10.1007/s11760-018-01410-3

30. Zhang L, Ren Z (2019) Comparison of CT and MRI images for the prediction of soft-tissue sarcoma grading and lung metastasis via a convolutional neural networks model. Clin Radiol 75:64-69 https://doi.org/10.1016/j.crad. 2019.08.008

\section{Publisher's Note}

Springer Nature remains neutral with regard to jurisdictional claims in published maps and institutional affiliations.

\section{Submit your manuscript to a SpringerOpen ${ }^{\circ}$ journal and benefit from:}

- Convenient online submission

- Rigorous peer review

- Open access: articles freely available online

- High visibility within the field

- Retaining the copyright to your article

Submit your next manuscript at $\boldsymbol{\nabla}$ springeropen.com 